\title{
A significant decrease of the fundamental Schumann resonance frequency during the solar cycle minimum of 2008-9 as observed at Modra Observatory
}

\author{
Adriena ONDRÁŠKOVÁ ${ }^{1}$, Sebastián ŠEVČÍK ${ }^{1}$, Pavel KOSTECKÝ ${ }^{1}$ \\ ${ }^{1}$ Department of Astronomy, Physics of the Earth and Meteorology, \\ Faculty of Mathematics, Physics and Informatics, Comenius University \\ Mlynská dolina F-1, 84248 Bratislava, Slovak Republic; \\ e-mail: adriena.ondraskova@fmph.uniba.sk
}

\begin{abstract}
The Schumann resonances (SR) are electromagnetic eigenmodes of the resonator bounded by the Earth's surface and the lower ionosphere. The SR frequency variability has been studied for more than 4 decades. Using data from the period 1988 to 2002, Sátori et al. (2005) showed that the SR fundamental mode frequency decreased on the 11-year time scale by $0.07-0.2 \mathrm{~Hz}$, depending on which component of the field was used for estimation and likely also on the location of the observer. A decrease by $0.30 \mathrm{~Hz}$ from the latest solar cycle maximum to the minimum of 2009 is found in data from Modra Observatory. This extraordinary fall of the fundamental mode frequency can be attributed to the unprecedented drop in the ionizing radiation in X-ray frequency band. Although the patterns of the daily and seasonal variations remain the same in the solar cycle minimum as in the solar cycle maximum, they are significantly shifted to lower frequencies during the minimum. Analysis of the daily frequency range suggests that the main thunderstorm regions during the north hemisphere summer are smaller in the solar cycle minimum than in the maximum.
\end{abstract}

Key words: electromagnetic field, ionosphere, Schumann resonances

\section{Introduction}

The natural electromagnetic radiation in the extremely low frequency (ELF) range is excited by the lightning activity in the three major regions: tropical Asia, Africa and America. Schumann (1952) theoretically predicted the existence of the resonant modes, called Schumann resonances (SR), due to 
constructive interference within the earth-ionosphere waveguide. The frequencies of these resonance modes are close to 8, 14, 20, $26 \mathrm{~Hz}$, etc.

Increasing interest in the SR in recent years can be attributed to the possible connection between the Earth's climate and the global lightning activity (Price, 1993). Moreover, Williams (1992) demonstrated some correlation between the first SR mode amplitude and the tropical land surface temperature anomaly on the El Niño - Southern Oscillation time scale. However, in order to use the SR measurements for studying the global lightning activity changes, it is necessary to understand the long-term variability of the natural SR signal.

The daily and seasonal variability of the SR parameters (peak frequency, relative amplitude and quality factor) of individual modes have been studied by e.g. Balser and Wagner (1962), Ogawa et al. (1969), Nickolaenko and Rabinowicz (1995), Sátori and Zieger (1996). However, most of these were for short periods. Sátori (1996) used data for more than 1 year. Price and Melnikov (2004) and Ondrášková et al. (2007) made use of 4-year measurements and analysed diurnal, seasonal and inter-annual variability of the three main SR parameters, but the interval of measurements was not long enough to resolve the 11-year variation.

Several studies documented that SR parameters vary on the 11-year time scale of the solar cycle (Sátori et al., 2000, 2005; Füllekrug et al., 2002; Kulak et al., 2003). The first mode frequency was found to decrease from the solar cycle maximum to minimum in $1996 / 1997$ by $0.07 \mathrm{~Hz}$ at Nagycenk determined from the electric field component, by $0.13 \mathrm{~Hz}$ at Arrival Heights in the Antarctic and by $0.2 \mathrm{~Hz}$ at Rhode Island (USA) from the magnetic field component (Sátori et al., 2005).

In this paper, we present results of measurements of the electric field SR component from October 2001 to May 2009 measured at Modra Observatory. This nearly 8 year interval is long enough to resolve the SR parameter variability on the 11-year time scale from the solar cycle maximum to the present solar cycle minimum, which appears to be extraordinary deep.

\section{Measuring equipment}

Monitoring of the vertical electric field SR component has been performed at the Astronomical and Geophysical Observatory (AGO) of Comenius Univer- 
sity near Modra, Slovakia ( $48.37^{\circ} \mathrm{N}, 17.27^{\circ} \mathrm{E}, 530 \mathrm{~m}$ a.s.l.). Since October 2001 there have been practically no interruptions except for the several cases when measuring hardware or software was being updated or short interruptions due to power outages or weather situation.

The electric field SR component is picked up by a capacitive (ball) antenna mounted on a $5 \mathrm{~m}$ insulator mast. The preamplifier (very high input impedance amplifier) is located immediately at the antenna base. $50-\mathrm{Hz}$ analog filters and three-channel 16 bit ADC follow. Details are given in Kostecký et al. (2000).

Since October 2001 through July 2006, the raw time series data were collected every half hour for 327.68 seconds (which is 65536 samples taken with $200 \mathrm{~Hz}$ sampling frequency), supplying 48 files per day. Since then data have been collected every 6 minutes, i.e. 240 files are stored per day.

\section{Data processing}

The data files are subjected to DFT. The raw SR spectra from Modra observatory (AGO) can be viewed in real time at http://193.87.5.150/. These spectra are then smoothed and subsequently fitted with 5 Lorentz-like functions of the form

$L_{i}(f)=\frac{A_{i}}{1+Q_{i}^{2}\left(\frac{f}{F_{i}}-\frac{F_{i}}{f}\right)^{2}}$,

where $F_{i}$ is the central (peak) frequency of the $i$-th mode, $A_{i}$ is the amplitude of the $i$-th mode, and $Q_{i}$ is the Q-factor, which is a dimensionless measure of the attenuation in the waveguide. More details about the fitting procedure and determination of the mode parameters are described in Rosenberg (2004). The first survey of the first four SR modes principal parameters for October 2001 -December 2005 is shown in Ondrášková et al. (2007).

\section{Daily frequency variations of $E_{z}$ component}

The obtained variations in frequency for the electric field component for the first SR mode are shown in Fig. 1, where there are graphs of diurnal 

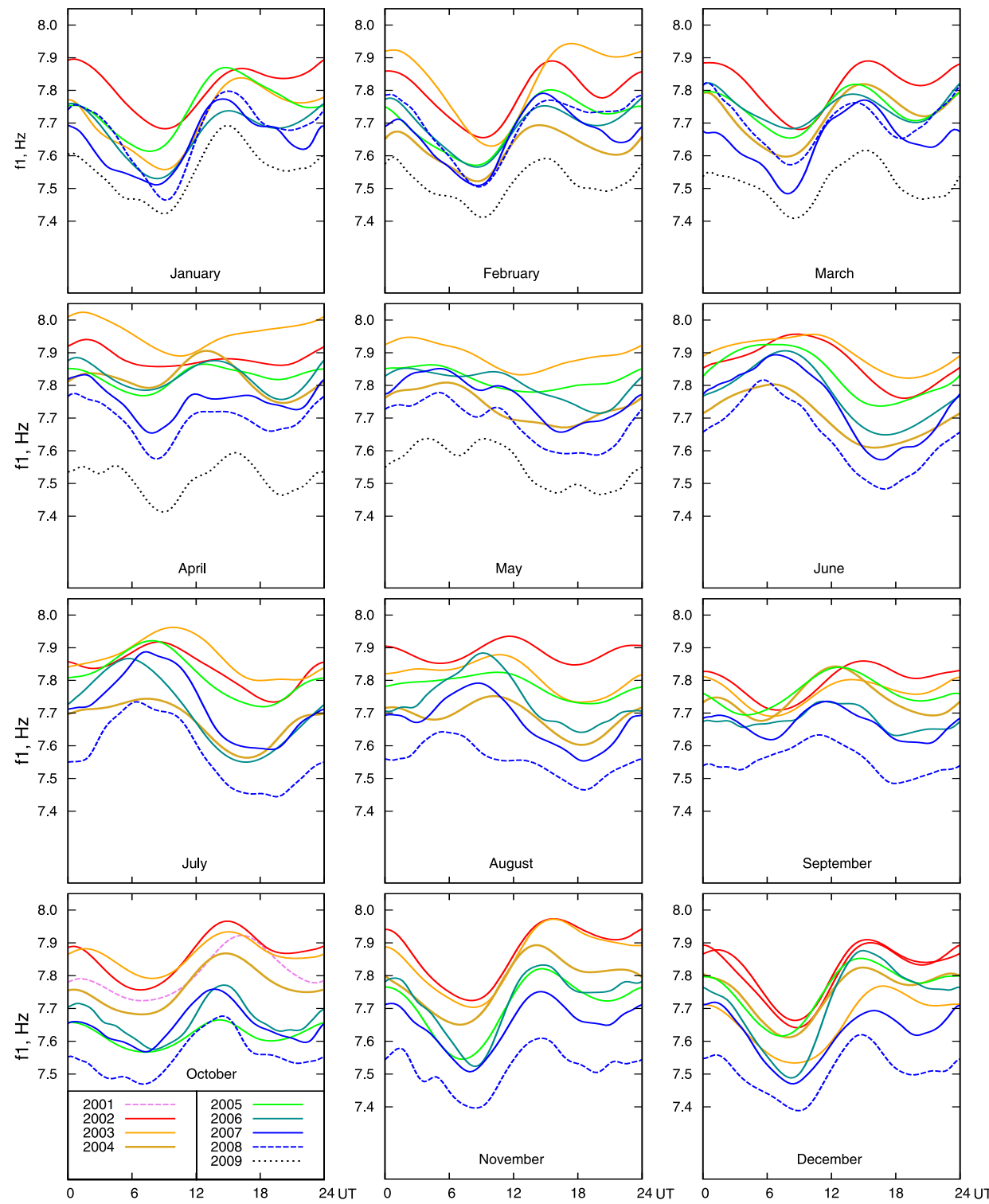

Fig. 1. Monthly averaged diurnal variations of the first-mode frequency in the years 2001-2009. Different bold lines are used for years of the solar cycle maximum, different thin lines for years of the solar cycle minimum. Colour scale appears in web-version. Note the analogical patterns in different years in all months. 
variation for every month of a year and for about 8 years of measurements. The diurnal frequency variation pattern of each mode is predominantly determined by the source-observer geometry (Sátori et al., 2003). The overall pattern in the whole year corresponds to the annual transition from the "winter-type" to the "summer-type" diurnal frequency variation and back to "winter-type" one.

The first mode shows only one primary maximum and one minimum in frequency, with their time varying with the season. In winter months (November-March) the minimum frequency occurs at about 9 UT at Modra, while in June-July the first mode frequency has its minimum in afternoon hours and maximum at around 9 UT.

Thanks to the proximity of the two observatories, our diurnal frequency variation curves are very similar to curves obtained at Nagycenk in 1993 1995 (Sátori and Zieger, 1996). On the other hand, a slight difference can be seen if our results are compared with the diurnal variation of the first mode frequency reported by Price and Melnikov (2004, last column in Fig. 4 therein), which is given as a 3-month average variation calculated from 4year averages. At least a part of the difference can be attributed to the different source-observer distance of the Mitzpe Ramon station in Israel. Secondly, comparison with the Price and Melnikov curves of the first mode diurnal variation is difficult for the fact that they show 3-month averages. As a consequence, the "September - October - November" curve mixes one month with typical "winter" pattern and one month with typical "transient" pattern. Similarly for the March - April - May curve.

In this paper we study the daily frequency variation for each month separately. Thus, it can be seen from Fig. 1 that the transition from the winter to the summer pattern occurs in some years in April and in other years in May. Curves for May have the summer pattern in 2007, 2008 and 2009. In June and July, all curves have the summer pattern, which persists in August in 2006, 2007 and 2008. This finding reveals a tendency that the summer-type daily variation lasts longer during the solar cycle minimum and shorter during the maximum.

The difference between the maximum and minimum frequencies is called a diurnal frequency range (DFR). It depends inversely on the size of the active thunderstorm region. Figure 1 shows that the first mode DFR in all studied years is maximal in November-January (about $0.25 \mathrm{~Hz}$ ) and this 
value is the same for years of the solar cycle maximum (2001-2004) as well as of the solar cycle minimum (2006-2009). The DFR is much narrower in the transition months April-May and August-September when it is sometimes as low as $0.1 \mathrm{~Hz}$.

The DFR in months with summer pattern daily variation (i.e. June, July) is $0.23 \mathrm{~Hz}$ on average. In fact, it rises from $0.17 \mathrm{~Hz}$ in $2002-2003$ to $0.30 \mathrm{~Hz}$ in $2006-2008$. This result suggests that the main thunderstorm regions during the north hemisphere summer are smaller in the solar cycle minimum and last from May to August.

\section{The 11-year variation}

As the geometry between the source and the observer approximately repeats every year it is understandable that the diurnal frequency variation curves for all years have nearly the same pattern. The interannual difference between the curves seen in Fig. 1 exhibits a systematic or quasi-systematic decrease of frequency from 2001-2 (year of the solar cycle maximum) to 2008-9 (year of the solar cycle minimum). The full bold curve is for 2001, the lowest thin dotted curve is for 2009. The average difference between the curve for 2002 and the curve for 2008 is $0.2-0.4 \mathrm{~Hz}$, see Fig. 1.

Figure 2 shows the daily mean values of the first mode frequency over the whole period of observations at Modra Observatory. Though the values reveal a significant day-to-day variability, which is superposed on 27-day, semiannual and annual variations, a systematic decrease of the frequency from the solar cycle maximum to minimum is evident.

The monthly and yearly (dotted lines) mean values of the fundamental mode frequency are depicted in Fig. 3. GOES satellite 5-minute averages of the solar X-radiation flux in both $0.1-0.8 \mathrm{~nm}$ and in $0.05-0.3 \mathrm{~nm}$ (http://goes.ngdc.noaa.gov/data/avg/) were used to compute monthly means and the results are plotted in Fig. 3, too. A deep minimum of this flux appeared from June 2008 up to May 2009. That is why all yearly means are calculated from June to May of the next year as indicated in Fig. 3. The yearly mean value of the X-ray flux in $0.1-0.8 \mathrm{~nm}$ decreased from $1.23 \times 10^{-6} \mathrm{Wm}^{-2}$ (June 2002 - May 2003) to $4.05 \times 10^{-9} \mathrm{Wm}^{-2}$ (June 2008 - May 2009). In the same period, the studied frequency went down 


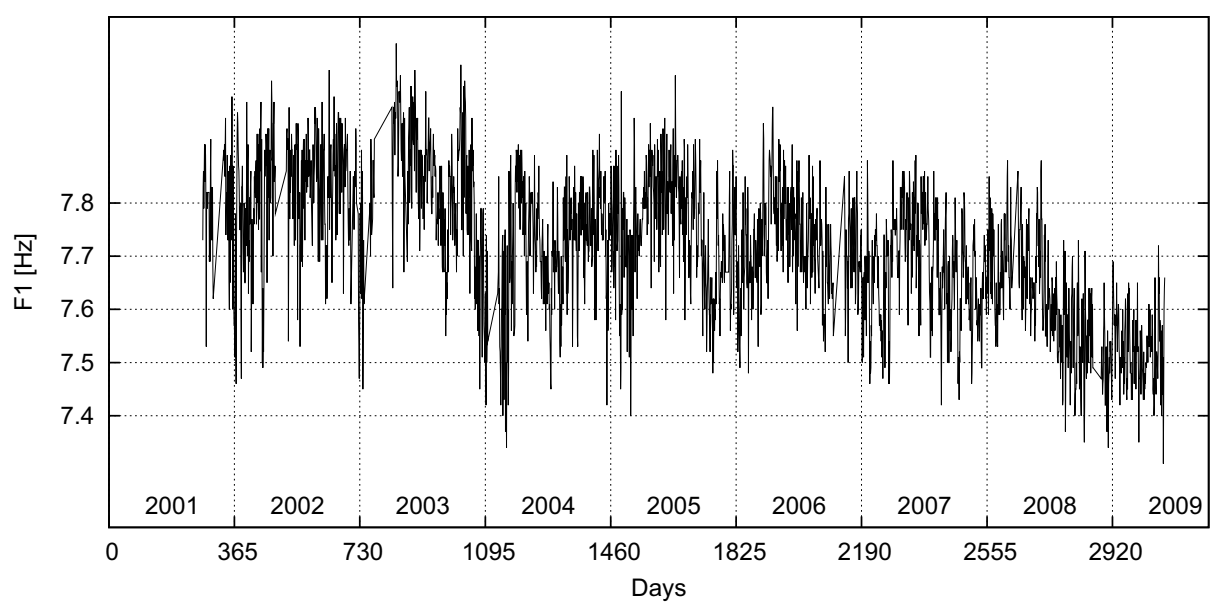

Fig. 2. The daily mean values of the fundamental Schumann resonance frequency from October 2001 up to May 2009. Note a significant decrease from the solar cycle maximum in 2001-2003 to the solar cycle minimum in 2008-2009.

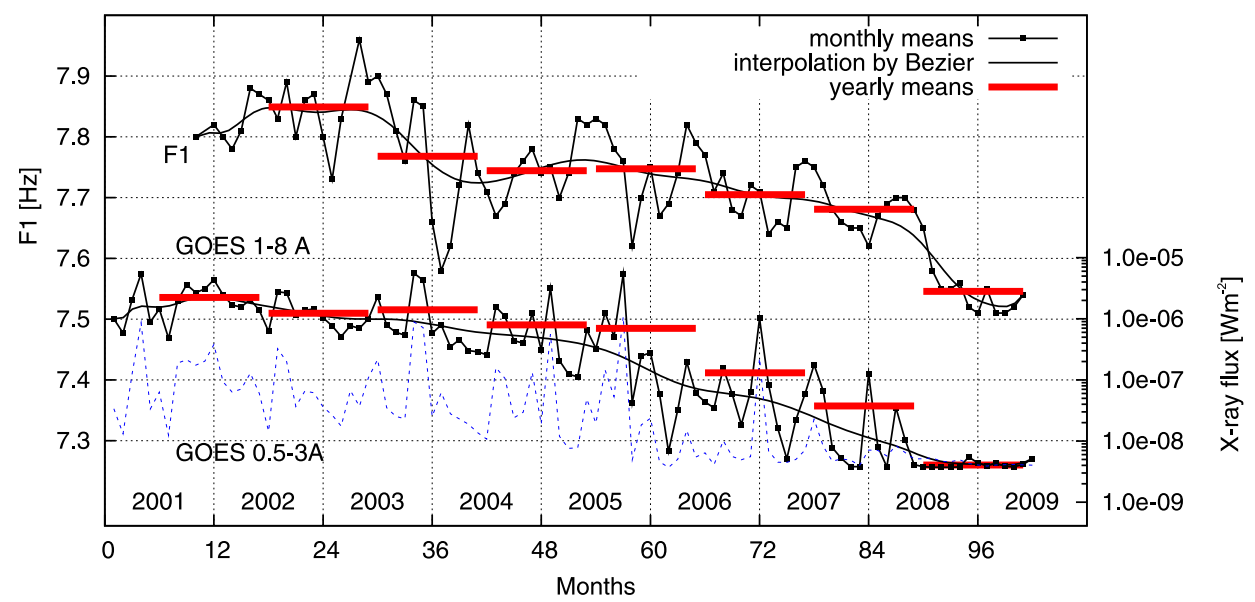

Fig. 3. The monthly and yearly mean values of the fundamental Schumann resonance frequency and X-ray flux in two frequency bands. A smooth full line is the Bezier interpolation. The yearly mean for June 2002 - May 2003 is $7.85 \mathrm{~Hz}$, the yearly mean for June 2008 - May 2009 is $7.55 \mathrm{~Hz}$. 
from $7.85 \mathrm{~Hz}$ (June 2002 - May 2003) to $7.55 \mathrm{~Hz}$ (June 2008 - May 2009). A smooth full line is the Bezier interpolation of Schumann fundamental frequency as well as the X-ray flux in $0.1-0.8 \mathrm{~nm}$ band.

The observed frequency decrease can be explained in terms of changes in the two characteristic layers, which represent the upper boundary of the waveguide, and which are responsible for ELF wave propagation. On the 11-year time scale, the solar output in X-ray range varies by 2 orders of magnitude, in the ultraviolet range by a factor of 2 and both are proportional to the solar activity. Cosmic rays, which are another source of ionization, also vary with the solar cycle but inversely. According to Sátori et al. (2005), changes of the $\mathrm{X}$-ray radiation dominate the variations in the conductivity profile within the upper characteristic layer (the $90-100 \mathrm{~km}$ portion of the E-region). The decrease of the this conductivity by up to one order of magnitude over the solar cycle is responsible for the observed SR frequency decrease by several tenths of $\mathrm{Hz}$ (Sátori et al., 2005), evaluated from SR magnetic component for the 2nd, 3rd and 4th modes.

\section{Conclusion}

Our observations support the findings of Sátori et al. (2005) that the variations of SR frequencies are in phase with the solar X-ray output over the solar cycle, i.e. over 11-year time scale. Our electric field component data show that the frequency decreased by $0.30 \mathrm{~Hz}$ from the latest solar maximum to the present minimum in 2009 . The obtained value is much greater than a value of $0.07 \mathrm{~Hz}$ deduced by Sátori et al. (2005) but for the previous solar cycle. The greater drop of frequency in this solar cycle minimum can be attributed to the very low level of solar activity and low level of solar output in X-ray range since June 2008, which is by one order of magnitude lower than during previous minimum of 1996/1997.

The level of the solar X-ray radiation (in $0.1-0.8 \mathrm{~nm}$ range) in the previous solar minimum was about $3 \times 10^{-8} \mathrm{Wm}^{-2}$. In the present solar minimum, this value was reached in 2007. In 2007, according to our observations, the yearly mean value of the 1st Schumann resonance frequency was $7.68 \mathrm{~Hz}$. It means that by that time the first SR mode decreased by $0.17 \mathrm{~Hz}$, the value similar to values obtained by Sátori et al. (2005) from the SR magnetic component data. 
Acknowledgments. This work was supported by the Grant No. 1/0521/08 of the Scientific Grant Agency VEGA.

\section{References}

Balser M., Wagner C. A., 1960: Observation of Earth-Ionosphere Cavity Resonances. Nature, 188, 638-641.

Füllekrug M., Fraser-Smith A. C., Schlegel K., 2002: Global ionospheric D-layer height monitoring. Europhysics Letters, 59, 626-629.

Kostecký P., Ondrášková A., Rosenberg L., Turňa L', 2000: Experimental setup for the monitoring of Schumann resonance electric and magnetic field variations at the Geophysical Observatory at Modra-Piesok. Acta Astron. et Geophys., XXI-XXII, 71-92.

Kulak A., Kubisz J., Michalec A., Ziba S., Nieckarz Z., 2003: Solar variations in extremely low frequency propagation parameters: 2. Observations of Schumann resonances and computation of the ELF attenuation parameter. J. Geophys. Res., 108 (A7), $1271 \mathrm{p}$.

Nickolaenko A. P., Rabinowitz L. M., 1995: Study of the annual changes of global lightning distribution and frequency variations of the first Schumann resonance mode. J. of Atmos. Terr. Phys., 57, 1345-1348.

Ogawa T., Tanaka Y., Yasuhara M., 1969: Schumann resonances and world-wide thunderstorm activity - diurnal variations of the resonant power of natural noise in the earth-ionosphere cavity. J. Geophys. Res., 21, 447-452.

Ondrášková A., Kostecký P., Ševčík S., Rosenberg L., 2007: Long-term observations of Schumann resonances at Modra Observatory, Radio Science, 42, RS2S09, doi : 10.10 29/2006RS003478.

Price C., 1993: Global surface temperatures and the atmospheric electrical circuit. Geophys. Res. Lett., 20, 1363-1366.

Price C., Melnikov A., 2004: Diurnal, seasonal and inter-annual variations in the Schumann resonance parameters. J. Atmos. Terr. Phys., 66, 1179-1185.

Rosenberg L., 2004: Data processing methodology of the electric and magnetic components of the Schumann resonances at Modra Observatory. Acta Astron. et Geophys., XXV, 1-8.

Sátori G., 1996: Monitoring Schumann resonances II. Daily and seasonal frequency variations. J. Atmos. Terr. Phys., 58, 1483-1488.

Sátori G., Zieger B., 1996: Spectral characteristics of Schumann resonances observed in Central Europe. J. Geophys. Res., 101, 29663-29669.

Sátori G., Williams E., Boccippio D. J., 2003: On the dynamics of the North-South Seasonal Migration of global Lightning. AGU, Fall Meeting 2003, abstract \#AE32A0167 . 
Sátori G., Williams E., Boldi R., Füllekrug M., 2000: Systematic variations of Schumann resonance frequencies on the 11-year solar cycle at multiple stations. Eos Transactions AGU, 81 (48), A12B-01.

Sátori G., Williams E., Mushtak V., 2005: Response of the Earth-ionosphere cavity resonator to the 11-year solar cycle in X-radiation. J. Atmos. Terr. Phys., 67, 553-562.

Schumann W. O., 1952: On the free oscillations of a conducting sphere which is surrounded by an air layer and a ionosphere shell. Zs. Naturforschung, 7a, 149-154 (in German).

Williams E. R., 1992: The Schumann resonance: a global tropical thermometer. Science, $256,1184-1187$. 